\title{
Dutch treat versus Oriental treat
}

\author{
Jeong-Yoo Kim ${ }^{\mathrm{a}, *}$, Hyung Bae ${ }^{\mathrm{a}}$, Dongchul Won ${ }^{\mathrm{b}}$ \\ ${ }^{a}$ Department of Economics, Dongguk University, 3-26 Pildong, Chungku, Seoul 100-715, South Korea \\ ${ }^{\mathrm{b}}$ Department of Industrial Information, Kongju National University, 527 Yesan, \\ Chungnan 340-800, South Korea
}

Received 2 June 2000; accepted 8 January 2001

\begin{abstract}
We introduce a class of goods called "ping-pong goods" whose values are generated only through joint consumption by two or more people. Mainly, we compare the efficiency of two fee sharing rules. One is the Dutch rule, whereby each participant bears its own expenses and the other is the Oriental rule, whereby the initial proposer bears all the expenses. We assert that the consumers' surplus under the Oriental rule is higher than the consumers' surplus under the Dutch rule only if the per-person price is high and the number of participants in consuming a ping-pong good is small. (C) 2002 Elsevier Science B.V. All rights reserved.
\end{abstract}

JEL classification: D69

Keywords: Ping-pong good; Dutch treat; Oriental treat; Fee-sharing rule

\section{Introduction}

There are many commodities whose values are generated only through joint consumption by two or more people. Phone calls, mailing, and chatting or playing a computer game through networks are typical examples. Playing sports games like ping-pong, tennis or baseball, and card games like poker or bridge are other examples. A date or a sex are also examples of commodities for which we need a partner. We will call such a commodity involving an extreme form of externalities a "ping-pong good" or a "participatory good". That is, a commodity is a ping-pong good if all the participating consumers can get positive utility only when a certain number (more than one) of the consumers participate in consuming the commodity.

An individual consumer's decision to participate in consuming a ping-pong good is usually made based on the share of the price he has to pay, not on the total price, so

\footnotetext{
* Corresponding author. Tel.: +82-2-2260-3716; fax: +82-2-2260-3716.

E-mail address: jyookim@dongguk.edu (J.-Y. Kim).
} 
that the demand for a ping-pong good is affected directly by the price-sharing rule among the participants. In a situation where externalities are involved like this, Coasian wisdom suggests that consumers may manage to consume an efficient amount of the commodity by voluntary negotiation among them if all the consumers' valuations for consuming the good are known and the negotiation costs are low enough. In reality, however, privacy of one's own valuation makes it difficult to reach an agreement in sharing the price and, instead, makes consumers resort to a pre-determined sharing rule. In the real world, there are two different sharing rules each of which seem to have become a social norm in the Western society and the Oriental society, respectively. One is for each participant to bear its own expenses and the other is for the initial proposer to bear all the expenses. The former convention is often called the Dutch treat (rule). In contrast, the latter convention will be called the Oriental treat (rule).

In this paper, we will compare the efficiency of the two sharing rules. Under the Oriental rule, not all the participants pay for consuming a ping-pong good, although it gives benefits to all of them. This seems to suggest that the Oriental rule is less efficient than the Dutch rule whereby everybody pays, but it is not necessarily the case. Essentially, the Dutch rule is more efficient if the price charged per person is high relative to the consumers' valuations in the sense that it can induce a higher participation rate of consumers, and thus, increase consumers' surplus, while the Oriental rule is more efficient when the price per person is relatively low so as to induce a high participation rate. The intuition is as follows. A person cannot consume a ping-pong good on his own. His consumption and the resultant realization of his valuation from the consumption occur only when the other agrees to consume it together. This implies that the other's decision generates externalities. Thus, a person may improve efficiency by giving a subsidy to the other. This is a possibility that may make the Oriental rule more efficient. However, such a subsidy makes it costly to initiate joint consumption for the proposer himself and lowers the likelihood that somebody proposes joint consumption. This is a possibility that may make the Oriental rule less efficient. The Oriental rule will be more efficient if the former positive effect of subsidizing the other dominates the latter negative effect. This is possible if the subsidy size (the per-person price) is small.

The ping-pong good is similar to the public good in the sense that all the participants consume the same amount of it. However, it is not exactly the same as the public good because the participants can exclude others from consuming it. It is also distinguished from the club good (Buchanan, 1965). They share the property of exclusiveness but the utility of each participant in consuming the ping-pong good is dependent on the participation of the other members whereas it is not usually expected in the consumption of the club good. That is, complementarity among participants is a salient feature of the ping-pong good. Also, the common feature that all the exclusive members consume the same amount is the property that is ex ante agreed cooperatively in the case of the ping-pong good, whereas it is the ex post consequence of each individual member's noncooperative decision making in the case of the club good. Thus, the amount consumed by the members is determined by the minimum that individual agents want to consume in the case of the ping-pong good, while it is determined by the maximum in the case of the club good. Furthermore, all the members do not necessarily consume the same amount of the club good if some particular member has a satiation point in his utility function. The ping-pong good also has some 
similarity to the good with network externalities in the sense that the participants' utilities are interdependent. But, in the case of the ping-pong good, the utility of consumers does not depend on the network size itself.

In Section 2, we introduce a simple model with only two players to illustrate when the Oriental rule can be more efficient than the Dutch rule. In Section 3, we provide a general model with many players and analyze it. Section 4 contains discussion and concluding remarks.

\section{The two-player model}

Consider a man (consumer 1) and a woman (consumer 2), thinking of going out for dinner together. Consumer $i$ values the date with consumer $j(j \neq i)$ at $v_{i}$ which is private information of consumer $i$. His or her valuation will be 0 if he or she does not date with the other. $v_{i}$ is uniformly distributed over $[0,1]$, and $v_{1}$ and $v_{2}$ are independent.

Now imagine that the activity of going out for dinner together is performed if either of the consumers proposes to do so and the other accepts the proposal. If both propose at the same time, the initiator is randomly determined with equal probability. The per-person price for a dinner is $p$.

Suppose that they go Dutch. Then, consumer $i$ proposes to go out for dinner if and only if $v_{i} \geq p$ and consumer $j$ accepts the proposal if and only if $v_{j} \geq p$. As a tie-breaking rule, we are assuming that indifference is always resolved in favor of going out.

On the other hand, the situation in the case of the Oriental treat is rather complicated. If somebody, say consumer $i$, proposes to go out together, it is always accepted since $v_{j} \geq 0$ for $j \neq i$. Then, when will a consumer propose to date? Suppose consumer $i$ proposes if and only if $v_{i} \geq v^{*}$. First, notice that $v^{*} \geq 2 p$, because the proposing strategy with $v^{*}<2 p$ would be dominated by not proposing at all. Then, notice that, if $p>1 / 2$, a date never happens under the Oriental rule, since neither will propose to date. We assume that $p<1 / 2$. To find $v^{*}$, we need to compute expected payoffs when a consumer proposes to date and when he does not. Denoting the probability distribution function of $v_{i}$ by $F(\cdot)$, the expected payoff that consumer $i$ with his valuation $v_{i}$ can get by not proposing a date is $\left[1-F\left(v^{*}\right)\right] v_{i}$ since the other consumer will propose to date with probability $1-F\left(v^{*}\right)$, and the expected payoff that he can get by proposing is $F\left(v^{*}\right)\left(v_{i}-2 p\right)+(1 / 2)\left[1-F\left(v^{*}\right)\right]\left\{\left(v_{i}-2 p\right)+v_{i}\right\}$ since the other consumer will not make a proposal with probability $F\left(v^{*}\right)$ and in this case passively accept the proposal of consumer $i$ and, if he also proposes, he will pay either $2 p$ or 0 with equal probability. Since $v^{*}$ is the valuation that makes consumer $i$ indifferent between proposing a date and not, it must satisfy

$$
\left[1-F\left(v^{*}\right)\right] v^{*}=F\left(v^{*}\right)\left(v^{*}-2 p\right)+\left[1-F\left(v^{*}\right)\right]\left(v^{*}-p\right) .
$$

Since we are assuming a uniform distribution for $v_{i}$ over $[0,1], F\left(v^{*}\right)$ is simply $v^{*}$, and thus, the equality is simplified to $\left(v^{*}\right)^{2}-p v^{*}-p=0$. From this, we get

$$
v^{*}=\frac{p+\sqrt{p^{2}+4 p}}{2},
$$

since $v^{*}>0$. By inspection, $v^{*}>2 p$, as required for the solution. Here, notice that a consumer with the valuation $2 p$ strictly prefers not to propose a date. This result arises from 


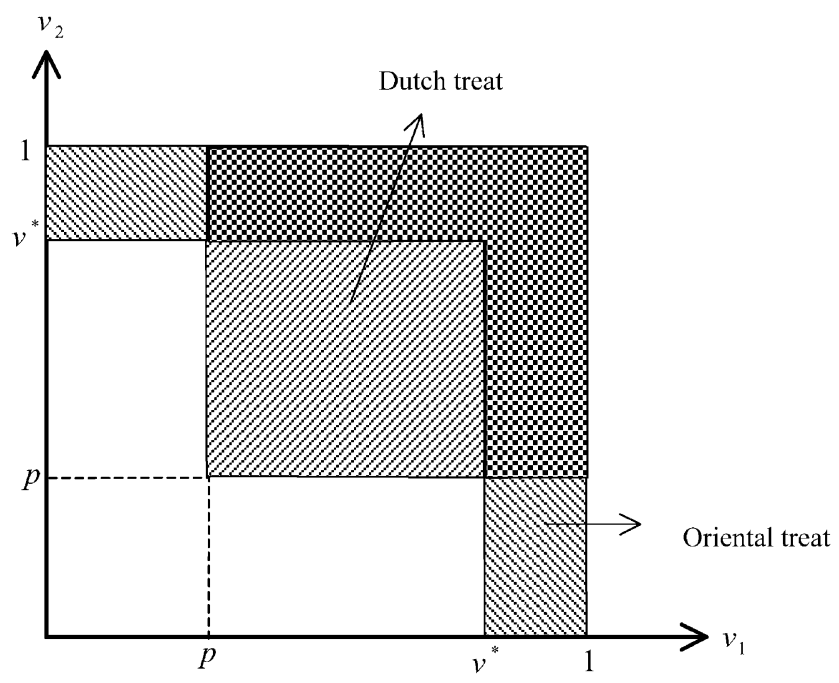

Fig. 1. Outcomes under Dutch rule and Oriental rule.

a strategic consideration. A consumer decides whether to propose a date or not by taking the possibility of free riding into account. A consumer does not need to think strategically in the Dutch rule, since he pays the same amount in the Dutch rule whether he is the proposer or the proposee. Fig. 1 depicts the outcomes under the Dutch rule and the Oriental rule. They date in the hatched area in each case.

Let us compare the probability of a date under the two systems. Under the Dutch rule, a date occurs with probability $P_{\mathrm{D}}=(1-p)^{2}$ and, under the Oriental rule, the probability is $P_{\mathrm{O}}=1-\left(v^{*}\right)^{2}$. Notice that the date probability is higher under the Oriental rule if and only if $p<1-(1 / \sqrt{2}) \approx 0.29$. This is intuitively clear. If $p=0$, the date occurs with certainty under either rule, implying that the date probability under each fee system is the same. As $p$ goes up, the probability under the Oriental rule is decreased to the second order while the probability under the Dutch rule is decreased to the first order. So, we can infer reasonably that the probability will be higher under the Oriental rule if $p$ is very low.

It is not difficult to see that both systems are far from efficient. Consumption would be efficient for all pairs $\left(v_{1}, v_{2}\right)$ such that $\left(v_{1}+v_{2}\right) \geq 2 p$. If $v_{1}$ and $v_{2}$ are known to both consumers, they can achieve the efficient outcome by agreeing to subsidize each other in such a way that they go out together if and only if $\left(v_{1}+v_{2}\right) \geq 2 p$. The efficient outcome is drawn in Fig. 2. In this example, too little consumption is made under both systems. Intuitively, consumption can occur under either system only when consumption is collectively rational, i.e. $\left(v_{1}+v_{2}\right) \geq 2 p$; otherwise, under either system, there must be someone who is reluctant to go out together.

It is worth comparing the consumers' surplus under the two systems. As shown in Appendix A, consumer surplus is $V_{\mathrm{D}}=(1-p)^{3}=(1-p) P_{\mathrm{D}}$ under the Dutch rule and $V_{\mathrm{O}}=(1-p)\left(1-\left(v^{*}\right)^{2}\right)=(1-p) P_{\mathrm{O}}$ under the Oriental rule. Thus, $V_{\mathrm{D}}>V_{\mathrm{O}}$ if and only if $P_{\mathrm{D}}>P_{\mathrm{O}}$. 


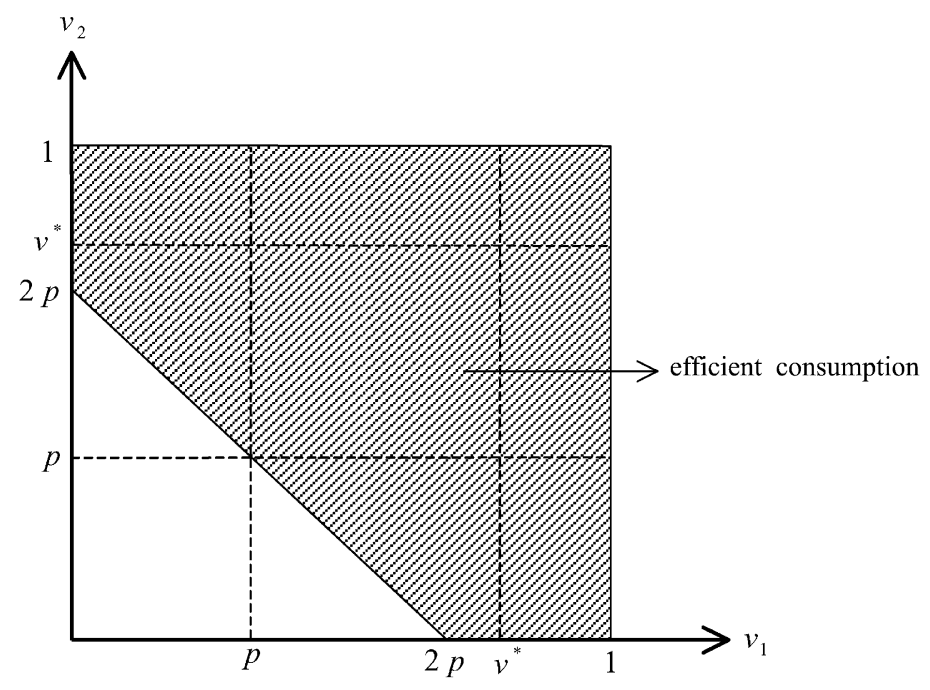

Fig. 2. Efficient outcome.

So far, we have implicitly assumed the symmetric equilibrium. In Appendix A, the proof is provided that there is no asymmetric equilibrium, so that the solution we found above is indeed the unique equilibrium.

\section{The $m$-player model}

We consider a ping-pong good requiring $m(\geq 2)$ players. A player proposes that the group of $m$ jointly consume the good. If more than one player proposes, one of the proposers is chosen, purely at random, to be the initial proposer. Responders individually respond by saying "yes" or "no". The $i$-th player has reservation price $v_{i}$ drawn independently from a common distribution function $F(\cdot)$. For now, $F(\cdot)$ is defined over any compact interval of the real line, though momentarily we will specialize to the uniform distribution. The reservation price for no activity is normalized at 0 , and the price per person is again $p$.

In the case of the Dutch treat, any player with $v_{i} \geq p$ will propose. After the proposal, a proposee $j$ accepts the proposal if and only if $v_{j} \geq p$. Therefore, the ping-pong good is consumed if $v_{i} \geq p$, for all $i$. In the case of the Oriental treat, suppose that any player with $v_{i} \geq v^{*}$ will make an initial proposal which is accepted by player $j$ with $v_{j} \geq 0$, that is, the ping-pong good is consumed if $v_{i} \geq v^{*}$ for some $i$ and $v_{j} \geq 0$ for all $j$. Notice that $v^{*}>m p$ is required in this case since no one would participate otherwise. Then, the probabilities of consumption under the two rules are

$$
\begin{aligned}
& P_{\mathrm{D}}=[1-F(p)]^{m}, \\
& P_{\mathrm{O}}=[1-F(0)]^{m}-\left[F\left(v^{*}(m, p)\right)-F(0)\right]^{m} .
\end{aligned}
$$


In words, $P_{\mathrm{D}}$ is the probability that $v_{i} \geq p$ for all $i$ and $P_{\mathrm{O}}$ is the probability that $v_{i} \geq 0$ for all $i$ and that $v_{i} \geq v^{*}$ for some $i$. Notice that $P_{\mathrm{D}}$ and $P_{\mathrm{O}}$ converge to 0 as $m$ is increased. Now, we have the following theorem.

Proposition 1. There exists a $p_{\epsilon}>0$ such that the probability that a ping-pong good is consumed under the Oriental rule is higher than the probability under the Dutch rule for all $p \in\left(0, p_{\epsilon}\right)$.

Proof. See Appendix A.

For the welfare analysis, we will, as in Section 2, assume that $F(v)$ is uniform over the interval $[0,1]$, implying that $F(v)=v$. Again let $V_{\mathrm{D}}$ and $V_{\mathrm{O}}$ denote the consumer surpluses. Then, we have

Lemma 1. (i) $V_{\mathrm{D}}=(m / 2)(1-p)^{m+1}$ and (ii) $V_{\mathrm{O}}=(m / 2)\left(1-\left(v^{*}\right)^{m+1}\right)-m p\left(1-\left(v^{*}\right)^{m}\right)$.

Proof. See Appendix A.

Lemma 2. Suppose there exists $v^{*} \in[0,1]$. Then, $\left(v^{*}\right)^{m}=p\left(\left(1-\left(v^{*}\right)^{m}\right)\right) /\left(\left(1-v^{*}\right)\right)$

Proof. See Appendix A.

Proposition 2. Suppose there exists $v^{*} \in[0,1]$. Then, $V_{\mathrm{D}}>V_{\mathrm{O}}$ if and only if $P_{\mathrm{D}}>P_{\mathrm{O}}$.

Proof. See Appendix A.

Now, we can establish our main theorems.

Theorem 1. (i) $V_{\mathrm{D}}>V_{\mathrm{O}}$ if $p \in(1 / m, 1)$ and (ii) $V_{\mathrm{O}}>V_{\mathrm{D}}$ if $p \in\left(0, p_{\epsilon}\right)$ for some $p_{\epsilon}>0$.

Proof. (i) Trivial since $V_{\mathrm{O}}=0$ and $V_{\mathrm{D}}>0$ if $p \in(1 / m, 1)$. (ii) Direct from Propositions 1 and 2.

Theorem 1 implies that consumers' surplus can be higher under the Oriental rule if $p$ is very low. Also, notice that the region within which the Oriental rule is more efficient disappears as the number of participants gets large. This implies that consumers' surplus is higher under the Dutch rule if the required number of participants is large enough.

\section{Discussion and conclusion}

In this paper, we have introduced the notion of the ping-pong good and discussed the relative efficiency of typical two fee sharing rules for the ping-pong good among participating members. The main result is that the Oriental rule can be more efficient only if the per-person price is low and the number of participants in consuming a ping-pong good is small. This may explain why the Oriental treat is usually observed only in such a limited situation. 
We will list some of restrictive features our model possesses. First, we have assumed that the valuations of the players are independent. In some cases, however, the valuations may be correlated. If they are perfectly correlated, for example, the Dutch rule will suffice to ensure efficiency. Second, we have implicitly assumed that consuming a ping-pong good requires $m$ designated members to take part in the activity. However, in many cases, it can also be launched if any arbitrary members of size $m$ in the whole population participate in the activity. Although our analysis has been focused on the case of designated members, the arguments so far can be extended straightforwardly to the case of arbitrary members. In this case, the probability that a ping-pong good is consumed under each system is summation of the probability that specific $m$ players are selected out of the whole population times the probability that a ping-pong good is consumed over all possible grouping cases, which turns out to be exactly the same as the probability obtained for the case of designated members. Therefore, Theorem 1 will be preserved for the case of arbitrary members. Third, we have focused only on two extreme forms of payment rules, but may consider a broader class of mechanisms for consumption decision and payment sharing for ping-pong goods. For example, players may adopt schemes, whereby a player bears some proportion (not necessarily a half) of the total price or gets a subsidy for participating in consuming a ping-pong good from the other. However, the problem of finding the optimal mechanism becomes essentially equivalent to that of public good provision. According to the results well-known in literature on mechanism design, neither Dutch treat nor Oriental treat will be optimal and the optimal mechanism will not be efficient in the sense that it cannot attain the first best outcome (see, for example, Myerson and Satterthwaite, 1983; Laffont and Maskin, 1979). Fourth, our analysis has been restricted to the partial equilibrium model. However, the existence of a ping-pong good will have some implications on the welfare property of the general equilibrium. In the presence of a ping-pong good, neither the Dutch treat nor the Oriental treat, in general, ensures the efficient allocation, since neither internalizes the externalities fully. The efficient allocation will be ensured by Lindahl pricing that is to differentiate the externality from the consumption that generates it and to treat it as a separate good.

Our model can be extended into various directions. First, we may consider a repeated game and see how repeated interaction between players can affect the relative efficiency of the Dutch treat and the Oriental treat. Second, we may incorporate a more realistic feature that people bred to the Dutch style and to the Oriental style are mingled in a society, instead of simply assuming that all the members of a society either pay in the Dutch style or pay in the Oriental style. Then, it will be an interesting issue which payment mode will dominate in the long run in an evolutionary perspective. Finally, we have not taken into account the supply side. If we explicitly consider suppliers that provide the ping-pong goods, new interesting issues will emerge. In particular, if a firm can set the price for a ping-pong good, its decision will surely be affected by the social convention of fee sharing. We look forward to seeing the fruitful enrichment of the model.

\section{Acknowledgements}

We are especially indebted to an anonymous referee who made many useful comments and constructive suggestions. We also wish to thank Jay Pil Choi, Yong-Gwan Kim, Youngse 
Kim and the audiences at the Economic Theory Workshop at Korea University. This work is supported by the Dongguk University research fund.

\section{Appendix A}

Derivation of $V_{\mathrm{D}}$ and $V_{\mathrm{O}}$ in Section 2

We have

$$
V_{\mathrm{D}}=\int_{p}^{1} \int_{p}^{1}\left(v_{1}+v_{2}-2 p\right) \mathrm{d} v_{1} \mathrm{~d} v_{2}=(1-p)^{3}=(1-p) P_{\mathrm{D}},
$$

since consumption is made under the Dutch rule it and only if $v_{i} \geq p$ for all $i=1,2$. Also, we have

$$
\begin{aligned}
V_{\mathrm{O}}= & \int_{v^{*}}^{1} \int_{0}^{1}\left(v_{1}+v_{2}-2 p\right) \mathrm{d} v_{1} \mathrm{~d} v_{2}+\int_{0}^{1} \int_{v^{*}}^{1}\left(v_{1}+v_{2}-2 p\right) \mathrm{d} v_{1} \mathrm{~d} v_{2} \\
& -\int_{v^{*}}^{1} \int_{v^{*}}^{1}\left(v_{1}+v_{2}-2 p\right) \mathrm{d} v_{1} \mathrm{~d} v_{2}=(1-p)\left(1-\left(v^{*}\right)^{2}\right)=(1-p) P_{\mathrm{O}},
\end{aligned}
$$

since consumption is made under the Oriental rule if and only if $v_{i} \geq v^{*}$ for some $i=1,2$.

Proof of nonexistence of asymmetric equilibria in Section 2.

Suppose consumer $i$ proposes if and only if $v_{i} \geq v_{i}^{*}$ in equilibrium. Then, $v_{1}^{*}$ and $v_{2}^{*}$ are characterized by

$$
\left[1-F\left(v_{j}^{*}\right)\right] v_{i}^{*}=F\left(v_{j}^{*}\right)\left(v_{i}^{*}-2 p\right)+\left[1-F\left(v_{j}^{*}\right)\right]\left(v_{i}^{*}-p\right), \quad i=1,2, \quad j \neq i,
$$

which are reduced to

$$
\begin{aligned}
& F\left(v_{2}^{*}\right)\left(v_{1}^{*}-p\right)=p, \\
& F\left(v_{1}^{*}\right)\left(v_{2}^{*}-p\right)=p .
\end{aligned}
$$

If $v_{1}^{*}=v_{a}, v_{2}^{*}=v_{b}$ is a solution, then, $v_{1}^{*}=v_{b}, v_{2}^{*}=v_{a}$ is also a solution. Substituting $v_{1}^{*}=v_{a}, v_{2}^{*}=v_{b}$ into (A.1) and $v_{1}^{*}=v_{b}, v_{2}^{*}=v_{a}$ into (A.2), we obtain $F\left(v_{b}\right)=F\left(v_{a}\right)$, which implies that $v_{a}=v_{b}$.

Proof of Proposition 1. Let $P_{\mathrm{D}}(p)$ and $P_{\mathrm{O}}(p)$ be the probability that the ping-pong good with its per-person price $p$ is consumed under each rule. Then, we have $P_{\mathrm{D}}(0)=P_{\mathrm{O}}(0)=$ $[1-F(0)]^{m}$. Let $\Phi(p) \equiv P_{\mathrm{O}}(p)-P_{\mathrm{D}}(p)$. Then, we have

$$
\frac{\partial \Phi}{\partial p}=m[1-F(p)]^{m-1} f(p)-m\left[F\left(v^{*}(p)\right)-F(0)\right]^{m-1} f\left(v^{*}(p)\right) \frac{\partial v^{*}}{\partial p},
$$

so that

$$
\frac{\partial \Phi(0)}{\partial p}=m[1-F(0)]^{m-1} f(0)>0 .
$$

Therefore, $\exists p_{\epsilon}>0$ such that, $\forall p \in\left(0, p_{\epsilon}\right), P_{\mathrm{O}}(p)>P_{\mathrm{D}}(p)$. 


\section{Proof of Lemma 1.}

$$
\begin{aligned}
V_{\mathrm{D}}= & \int_{p}^{1} \cdots \int_{p}^{1}\left(v_{1}+\cdots+v_{m}-m p\right) \mathrm{d} v_{1} \cdots \mathrm{d} v_{m} \\
= & \frac{m}{2}(1-p)^{m}(1+p)-m p(1-p)^{m}=\frac{m}{2}(1-p)^{m+1} \\
V_{\mathrm{O}}= & \int_{0}^{1} \cdots \int_{0}^{1}\left(v_{1}+\cdots+v_{m}-m p\right) \mathrm{d} v_{1} \cdots \mathrm{d} v_{m} \\
& -\int_{0}^{v^{*}} \cdots \int_{0}^{v^{*}}\left(v_{1}+\cdots+v_{m}-m p\right) \mathrm{d} v_{1} \cdots \mathrm{d} v_{m} \\
= & \frac{m}{2}-m p-\frac{m}{2} v\left(v^{*}\right)^{m}\left(v^{*}-2 p\right)=\frac{m}{2}\left(1-\left(v^{*}\right)^{m+1}\right)-m p\left(1-\left(v^{*}\right)^{m}\right)
\end{aligned}
$$

Proof of Lemma 2. Since every player is symmetric, it is enough to prove the result for consumer 1 . By definition of $v^{*}$, it must satisfy the equation

$$
\begin{aligned}
& P\left(\min \left\{v_{j}, j \neq 1\right\} \geq v^{*}\right) v^{*} \\
&= P\left(\max \left\{v_{j}, j \neq 1\right\}<v^{*}\right)\left(v^{*}-m p\right) \\
&+\frac{C_{1}^{m-1}}{2} P\left(v_{2} \geq v^{*}, v_{3} \geq v^{*}, v_{4}<v^{*}, \ldots, v_{m}<v^{*}\right) \\
& \times\left(2 v^{*}-m p\right) \ldots \frac{C_{m-1}^{m-1}}{m} P\left(v_{2} \geq v^{*}, v_{3} \geq v^{*}, \ldots, v_{m} \geq v^{*}\right)\left(m v^{*}-m p\right)
\end{aligned}
$$

The number, $C_{j}^{m-1}(j=1, \ldots, m-1)$ denotes a combination of $j$ players among $m-1$ players except for player 1 whose valuations are supposed to be higher than $v^{*}$. It is fair to treat only the probability that the first $j$ persons become a proposer because of the symmetry among players. The left-hand side of the equality in the above relation is equal to $\left(1-F\left(v^{*}\right)^{m-1}\right) v^{*}=v^{*}-\left(v^{*}\right)^{m}$. Its right-hand side has form

$$
\begin{aligned}
F\left(v^{*}\right)^{m-1}\left(v^{*}-m p\right) & +\frac{C_{1}^{m-1}}{2}\left[1-F\left(v^{*}\right)\right] F\left(v^{*}\right)^{m-2}\left(2 v^{*}-m p\right)+\cdots \\
& +\frac{C_{m-1}^{m-1}}{m}\left[1-F\left(v^{*}\right)\right]^{m-1}\left(m v^{*}-m p\right) \\
= & v^{*}\left\{F\left(v^{*}\right)^{m-1}+C_{1}^{m-1}\left[1-F\left(v^{*}\right)\right] F\left(v^{*}\right)^{m-2}+\cdots\right. \\
& \left.+C_{m-1}^{m-1}\left[1-F\left(v^{*}\right)\right]^{m-1}\right\}-p\left\{C_{1}^{m} F\left(v^{*}\right)^{m-1}\right. \\
& \left.+C_{2}^{m}\left[1-F\left(v^{*}\right)\right] F\left(v^{*}\right)^{m-2}+\cdots+C_{m}^{m}\left[1-F\left(v^{*}\right)\right]^{m-1}\right\} \\
= & v^{*}\left[F\left(v^{*}\right)+1-F\left(v^{*}\right)\right]^{m-1}-\frac{p}{1-F\left(v^{*}\right)} \\
& \times\left\{\left[F\left(v^{*}\right)+1-F\left(v^{*}\right)\right]^{m}-F\left(v^{*}\right)^{m}\right\} \\
= & v^{*}-p \frac{1-F\left(v^{*}\right)^{m}}{1-F\left(v^{*}\right)}=v^{*}-p \frac{1-\left(v^{*}\right)^{m}}{1-v^{*}}
\end{aligned}
$$

The result is followed by equating the outcomes of both sides. 
Proof of Proposition 2. It is straightforward to see that $V_{\mathrm{D}}=(m / 2)(1-p) P_{\mathrm{D}}$.

On the other hand, rearranging the result of lemma 2, we obtain $\left(v^{*}\right)^{m+1}=\left(v^{*}\right)^{m}-$ $p\left(1-\left(v^{*}\right)^{m}\right)$. Substituting it into $V_{\mathrm{O}}$, we see

$$
\begin{aligned}
V_{\mathrm{O}} & =\frac{1}{2} m\left[1-\left(v^{*}\right)^{m}+p\left(1-\left(v^{*}\right)^{m}\right)\right]-m p\left(1-\left(v^{*}\right)^{m}\right) \\
& =\frac{1}{2} m(1-p)\left(1-\left(v^{*}\right)^{m}\right)=\frac{1}{2} m(1-p) P_{\mathrm{O}} .
\end{aligned}
$$

\section{References}

Buchanan, J.M., 1965. An economic theory of clubs. Economica 32, 1-14.

Laffont, J.-J., Maskin, E., 1979. A differential approach to expected utility maximising mechanisms. In: Laffont, J.-J. (Ed.), Aggregation and Revelation of Preferences. North-Holland, Amsterdam.

Myerson, R., Satterthwaite, M., 1983. Efficient mechanisms for bilateral trading. Journal of Economic Theory $28,265-281$. 